\title{
Synthesis of $N$-protected Nortopsentins B and D
}

\author{
Christopher J. Moody ${ }^{\mathrm{a}, \mathrm{b}^{*}}$ and Jonathan R. A. Roffey ${ }^{\mathrm{a}}$ \\ ${ }^{a}$ Department of Chemistry, Loughborough University, Loughborough, Leicestershire, \\ LE11 3TU, UK \\ ${ }^{\mathrm{b}}$ School of Chemistry, University of Exeter, Stocker Road, Exeter, EX4 4QD, UK \\ E-mail: C.J.Moody@exeter.ac.uk
}

Dedicated to Professor Gurnos Jones on his $7^{\text {th }}$ birthday

(receive 10d Mar 00; accepted 20 Aug 00; published on the web 28 Aug 00)

\begin{abstract}
Indole-3-carboxamidines 13 and 14, prepared from the corresponding amides 7 and 8 via the thioamides 9 and 10, were reacted with 3-bromoacetylindole 15 to give the 2,4diindolylimidazoles 20 and 21, $N$-protected nortopsentins $\delta$ and B.
\end{abstract}

Keywords: Nortopsentin synthesis, indole-3-carboxamidines, thioamides

\section{Introduction}

Nortopsentins A-C 1-3, bis(indolyl)imidazoles isolated from the deep sea sponge Spongosorites ruetzler, are reported to have cytotoxic and antifungal properties. ${ }^{1}$ The debrominated derivative nortopsentin $\delta 4$ was formed by catalytic hydrogenolysis of the brominated compounds. The natural products have been synthesized by palladium( 0$)$ catalysed coupling of 3-indolylboronic acids with appropriate halogenated imidazoles, ${ }^{2}, 3$ and recently the corresponding bis(indolyl)thiazole analogues have been prepared. ${ }^{4}$ In view of our own interest in 3-indolyl substituted heterocycles (thiazoles and oxazoles), ${ }^{5-8}$ we undertook a different approach to the nortopsentins based on the reaction of a-haloketones with amidines.

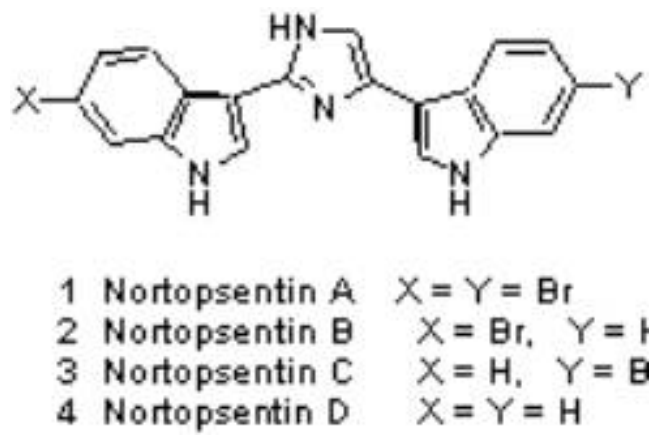




\section{Results and Discussion}

The synthesis of imidazoles based on the reaction of amidines with a-halo-carbonyl compounds is analogous to the Hantzsch synthesis of thiazoles which uses thioamides in place of amidines. Therefore the proposed route to the nortopsentins required the synthesis of the indole-3carboxamidines 13 and 14, protected as their $N$-Boc derivatives, and the 3-bromoacetyl indoles 15 and 19. The starting materials for the preparation of the indole-3-carboxamidines 13/14 were the corresponding carboxamides $7 / 8$ (Scheme 1). The amides were conveniently prepared by reaction of the corresponding indoles $5 / 6$ with chlorosulfonyl isocyanate (CSI) followed by hydrolysis of the chlorosulfonyl group, ${ }^{9}$ the electron withdrawing nature of the tertbutoxycarbonyl group not preventing the reaction with the highly electrophilic isocyanate. Reaction of the amides 7/8 with Lawesson's reagent (LR) in boiling benzene gave the corresponding thioamides $9 / 10$ which were converted into the thioimidates $11 / 12$ by alkylation with iodomethane. Finally ammonolysis of the thioimidates gave the required indole-3carboxamidines 13 and 14 (Scheme 1) as their hydrochlorides.

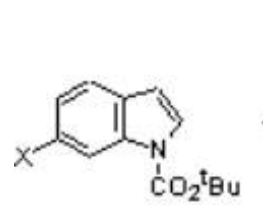

$5 X=H$

$$
6 \mathrm{X}=\mathrm{Br}
$$

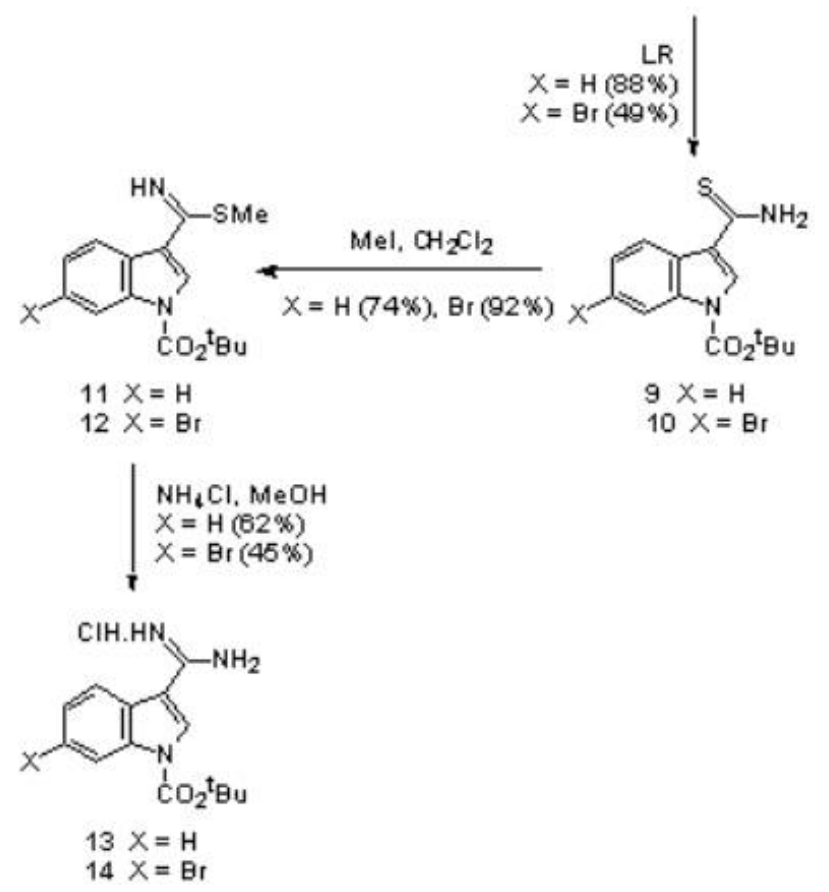

\section{Scheme 1}


Two strategies were employed to prepare the required 3-bromoacetylindoles. The 6unsubstituted compound 15 was prepared as previously described ${ }^{7}$ by acylation of indole with chloroacetyl chloride, ${ }^{10}$ followed by halide exchange (Scheme 2). The $N$-Boc-6-bromo derivative 19 was prepared differently. Thus Vilsmeier acetylation of 6-bromoindole followed by $N$ protection gave the 3-acetyl-6-bromoindole 18 in modest yield over the two steps. Bromination of the ketone using copper (II) bromide ${ }^{11}$ gave the required 3-bromoacetylindole 19 (Scheme 2).

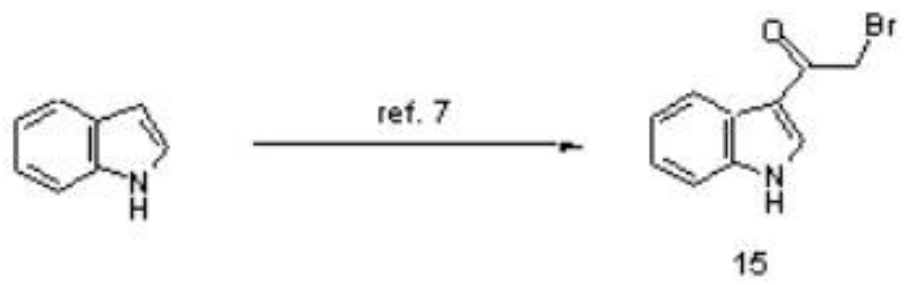

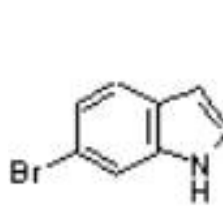

16

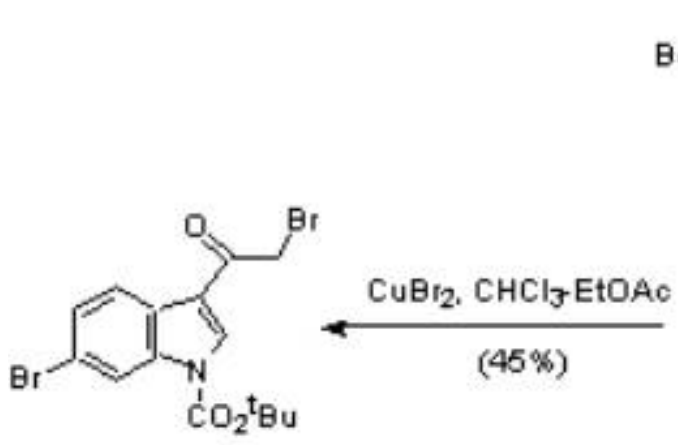

19

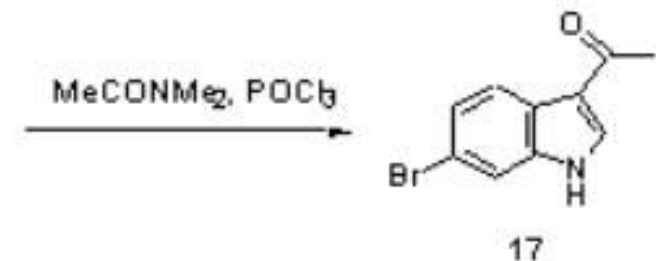

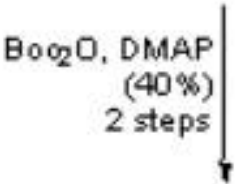

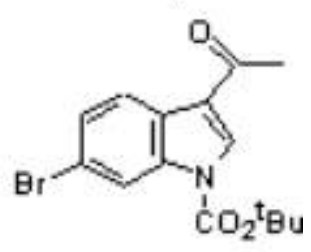

18

\section{Scheme 2}

With both the indole-3-carboxamidines and the bromoketones in hand the stage was set for the key imidazole forming reactions. Thus reaction of the indole-3-carboxamidine 13 with 3bromoacetylindole 15 in acetonitrile in the presence of potassium carbonate gave the bis(indolyl)imidazole 20 in $41 \%$ yield. Similarly, reaction of the 6-bromoindole-3carboxamidine 14 with bromoketone 15 gave the bis(indolyl)imidazole 21 (34\%) (Scheme 3). However, attempts to carry out similar reactions on the 6-bromo-3-bromoacetylindole 19 were unsatisfactory and attempts to isolate and identify the desired bis(indolyl)imidazoles 22 and 23, $N$-protected nortopsentins $\mathrm{C}$ and A respectively, proved fruitless. 


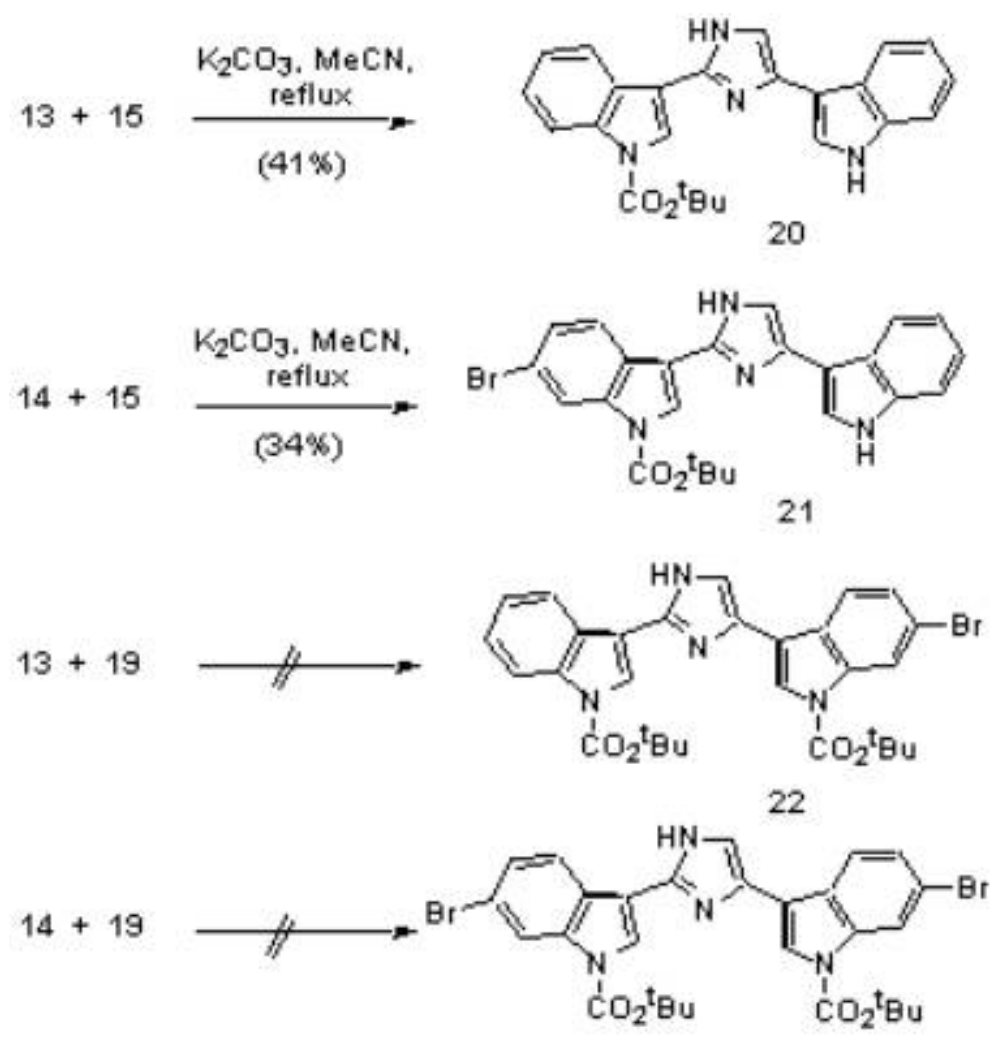

23

\section{Scheme 3}

With the $N$-Boc derivatives of nortopsentins $\delta$ and B in hand, efforts were made to deprotect the compounds to give the natural products. However, attempts to removed the Boc groups under the usual acidic conditions (TFA) or using sodium methoxide both failed to give the bis(indolyl)imidazoles 2 and 4. Under both sets of conditions, bright blue solutions were formed, concentration of which failed to give any recognisable products. Hence it would appear that the nortopsentins are unstable to acidic and basic conditions (or are readily oxidized); previous syntheses employed silyl protecting groups on nitrogen which were removed under neutral conditions., 3 It is interesting that in the original paper describing the isolation of the nortopsentins it was noted that the compounds became coloured on exposure to light hence requiring isolation and purification in dimly lit rooms.

In conclusion we have shown that simple two-component coupling of indole-3carboxamidines with 3-bromoacetylindoles can provide a route to bis(indolyl)imidazoles, although the reaction apparently fails when both indole nitrogens are protected with tertbutoxycarbonyl groups. 


\section{Experimental Section}

General Procedures. For general experimental details, see refs. 5 and 7. Compounds characterised by high resolution mass spectrometry were chromatographically homogeneous, and judged pure by analysis of their high field ${ }^{13} \mathrm{C}$ NMR spectra.

1-tert-Butoxycarbonylindole-3-carboxamide (7). CSI (7 g, $40.8 \mathrm{mmol}$ ) was added dropwise to a solution of tert-butyl indole-1-carboxylate $5(8.89 \mathrm{~g}, 40.8 \mathrm{mmol})$, and acetonitrile $(50 \mathrm{~mL})$ over a $10 \mathrm{~min}$ period at $0{ }^{\circ} \mathrm{C}$. The mixture was warmed to $\mathrm{rt}$ and stirred for $1 \mathrm{~h}$. A solution of acetone $(40 \mathrm{~mL})$ and water $(5 \mathrm{~mL})$ was added and the solution was rendered alkaline by the addition of potassium hydroxide (10\% solution, approx. $100 \mathrm{~mL})$. The mixture was extracted with ethyl acetate $(3 \times 50 \mathrm{~mL})$ and the extracts were combined and washed with brine $(50 \mathrm{~mL})$, dried $\left(\mathrm{MgSO}_{4}\right)$ and evaporated under reduced pressure. Recrystallization (dichloromethane) gave the title compound $\left(6.85 \mathrm{~g}, 64 \%\right.$ ) as a colorless solid, mp 163-164 ${ }^{\circ} \mathrm{C}$; (Found: $\mathrm{M}^{+}, 260.1180$. $\mathrm{C}_{14} \mathrm{H}_{16} \mathrm{~N}_{2} \mathrm{O}_{3}$ requires $\left.\mathrm{M}, 260.1161\right) ; v_{\max }\left(\mathrm{CHCl}_{3}\right) / \mathrm{cm}^{-1} 1740,1666,1450,1215,1153,768$; ${ }^{1} \mathrm{HNMR} \delta\left(250 \mathrm{MHz} ; \mathrm{CDCl}_{3}\right) 1.69\left(9 \mathrm{H}, \mathrm{s},\left(\mathrm{CH}_{3}\right)_{3}\right), 5.80(2 \mathrm{H}, \mathrm{br}, \mathrm{NH}), 7.36(2 \mathrm{H}, \mathrm{m}), 8.07(1 \mathrm{H}$, $\mathrm{d}, J=8.2 \mathrm{~Hz}), 8.13(1 \mathrm{H}, \mathrm{m}), 8.21(1 \mathrm{H}, \mathrm{d}, J=8.2 \mathrm{~Hz}) ;{ }^{13} \mathrm{CNMR} \delta\left(62.5 \mathrm{MHz} ; \mathrm{CDCl}_{3}\right) 31.6$ $\left(\left(\mathrm{CH}_{3}\right)_{3}\right), 88.9\left(\mathrm{C}_{\left.\left(\mathrm{CH}_{3}\right)_{3}\right)}\right), 118.9,125.1,127.3,127.5,128.8,131.5,132.6,139.1,153.7,171.8$; $\mathrm{m} / \mathrm{z} 260\left(\mathrm{M}^{+}, 10 \%\right), 204$ (20), 160 (30), 144 (30), 57 (100), 41 (30).

1-tert-Butoxycarbonylindole-3-thiocarboxamide (9). 1-tert-Butoxycarbonylindole-3-carboxamide 7 (2.00 g, $7.69 \mathrm{mmol})$, Lawesson's reagent $(1.55 \mathrm{~g}, 3.84 \mathrm{mmol})$ and benzene $(20 \mathrm{~mL})$ were heated at reflux for $1 \mathrm{~h}$. The reaction mixture was cooled and evaporated under reduced pressure to give a yellow solid. Flash column chromatography (dichloromethane : diethyl ether elution) gave the title compound (1.88 $\mathrm{g}$, 90\%) as a yellow solid, mp 98-99 ${ }^{\circ} \mathrm{C}$; (Found: $\mathrm{M}^{+}$, 276.0922. $\mathrm{C}_{14} \mathrm{H}_{16} \mathrm{~N}_{2} \mathrm{O}_{2} \mathrm{~S}$ requires $\mathrm{M}$, 276.0932); $v_{\max }\left(\mathrm{CHCl}_{3}\right) / \mathrm{cm}^{-1} 1742,1598,1372,1215,755 ;{ }^{1} \mathrm{HNMR}$ d $\left(250 \mathrm{MHz} ; \mathrm{CDCl}_{3}\right) 1.68$ $\left(9 \mathrm{H}, \mathrm{s},\left(\mathrm{CH}_{3}\right)_{3}\right), 7.34(2 \mathrm{H}, \mathrm{m}), 7.86\left(2 \mathrm{H}, \mathrm{br}, \mathrm{NH}_{2}\right), 8.03(1 \mathrm{H}, \mathrm{d}, J=8.2 \mathrm{~Hz}), 8.19(1 \mathrm{H}, \mathrm{d}, J=8.2$ $\mathrm{Hz}), 8.25(1 \mathrm{H}, \mathrm{s}) ;{ }^{13} \mathrm{CNMR} \delta\left(62.5 \mathrm{MHz} ; \mathrm{CDCl}_{3}\right)$ 28.0, 85.3, 115.6, 120.4, 121.0, 124.0, 125.2, 126.0, 128.5, 129.7, 135.8, 159.9; m/z $276\left(\mathrm{M}^{+}, 5 \%\right), 142$ (20), 84 (65), 57 (30), 49 (100), 41 (20), 31 (20).

Methyl 1-tert-butoxycarbonylindole-3-thiocarboximidate (11). 1-tert-Butoxycarbonylindole3-thiocarboxamide $9(1.20 \mathrm{~g}, 4.34 \mathrm{mmol})$, methyl iodide $(1.86 \mathrm{~g}, 13.2 \mathrm{mmol})$ and dichloromethane $(10 \mathrm{~mL})$ were stirred at $\mathrm{rt}$ for $48 \mathrm{~h}$ under a nitrogen atmosphere. Dichloromethane $(30 \mathrm{~mL})$ was addded and the mixture was washed with a saturated solution of sodium hydrogen carbonate $(2 \times 20 \mathrm{~mL})$, brine $(2 \times 20 \mathrm{~mL})$, dried $\left(\mathrm{MgSO}_{4}\right)$ and evaporated under reduced pressure to give the title compound( $0.93 \mathrm{~g}, 74 \%$ crude) as yellow solid which was used in the next step without further purification; $v_{\max }\left(\mathrm{CHCl}_{3}\right) / \mathrm{cm}^{-1} 1741,1653,1451,1215$, 757; ${ }^{1} \mathrm{HNMR}\left(250 \mathrm{MHz} ; \mathrm{CDCl}_{3}\right) \delta 1.69\left(9 \mathrm{H}, \mathrm{s},\left(\mathrm{CH}_{3}\right)_{3}\right), 2.46(3 \mathrm{H}, \mathrm{s}, \mathrm{SCH}), 3.50(1 \mathrm{H}, \mathrm{br}, \mathrm{NH})$, $7.34(2 \mathrm{H}, \mathrm{m}), 8.07(1 \mathrm{H}, \mathrm{s}), 8.17(2 \mathrm{H}, \mathrm{m})$.

1-tert-Butoxycarbonylindole-3-carboxamidine hydrochloride (13). Methyl 1-tertbutoxycarbonylindole-3-thiocarboximidate 11 (1.13 g, $3.9 \mathrm{mmol})$, ammonium chloride (2.17 g, 
$4.12 \mathrm{mmol})$ and methanol $(8 \mathrm{~mL})$ were heated under reflux for $3 \mathrm{~h}$. CAUTION: during the reaction methyl mercaptan is liberated and so a potassium permanganate bubbler was attached to the reflux condenser. The reaction mixture was cooled and ethyl acetate $(100 \mathrm{~mL})$ was added. The mixture was washed with brine $(2 \times 30 \mathrm{~mL})$, dried $\left(\mathrm{MgSO}_{4}\right)$ and evaporated under reduced pressure to give a pale brown solid. Crystallization (ethanol) gave the title compound $(0.71 \mathrm{~g}$, $62 \%$ ) as a pale brown solid, $\mathrm{mp} 207-208{ }^{\circ} \mathrm{C}$, used without further purification; ${ }^{1} \mathrm{HNMR} \delta(250$ $\left.\mathrm{MHz} ; \mathrm{CDCl}_{3}\right) 1.61\left(9 \mathrm{H}, \mathrm{s}, \mathrm{C}\left(\mathrm{CH}_{3}\right)_{3}\right), 7.26(2 \mathrm{H}, \mathrm{m}), 7.47(1 \mathrm{H}, \mathrm{m}), 8.03(1 \mathrm{H}, \mathrm{m}), 8.39(2 \mathrm{H}, \mathrm{br}$, $\left.\mathrm{NH}_{2}\right), 8.78(1 \mathrm{H}, \mathrm{s}), 9.31(1 \mathrm{H}, \mathrm{br}, \mathrm{NH})$.

tert-Butyl 6-bromoindole-1-carboxylate (6). 6-Bromoindole (2.00 g, $10.25 \mathrm{mmol})$, di-tert-butyl dicarbonate $(2.46 \mathrm{~g}, 11.28 \mathrm{mmol})$, DMAP $(122 \mathrm{mg}, 1 \mathrm{mmol})$ and acetonitrile $(28 \mathrm{~mL})$ were stirred at $\mathrm{rt}$ for $1 \mathrm{~h}$. Saturated sodium hydrogen carbonate solution $(20 \mathrm{~mL})$ was added and the mixture stirred for a further $30 \mathrm{~min}$. The mixture was extracted with diethyl ether $(2 \mathrm{x} 20 \mathrm{~mL})$ the extracts were combined washed with brine $(2 \times 20 \mathrm{~mL})$, dried $\left(\mathrm{MgSO}_{4}\right)$ and evaporated under reduced pressure. Flash column chromatography (light petroleum : diethyl ether elution) gave the title compound $(2.34 \mathrm{~g}, 77 \%)$ as a colorless solid, mp 60-62 ${ }^{\circ} \mathrm{C}$; (Found: $\mathrm{M}^{+}, 295.0216 . \mathrm{C}_{13} \mathrm{H}_{14}{ }^{79}$ $\mathrm{BrNO}_{2}$ requires M, 295.0208); $v_{\max }\left(\mathrm{CHCl}_{3}\right) / \mathrm{cm}^{-1} 1732,1434,1377,1215,761 ;{ }^{1} \mathrm{HNMR} \delta(250$ $\left.\mathrm{MHz} ; \mathrm{CDCl}_{3}\right) 1.69\left(9 \mathrm{H}, \mathrm{s},\left(\mathrm{CH}_{3}\right)_{3}\right), 6.54(1 \mathrm{H}, \mathrm{d}, J=3.7 \mathrm{~Hz}), 7.33(1 \mathrm{H}, \mathrm{dd}, J=8.31 .7 \mathrm{~Hz}), 7.41$ $(1 \mathrm{H}, \mathrm{d}, J=8.3 \mathrm{~Hz}), 7.56(1 \mathrm{H}, \mathrm{d}, J=3.7), 8.37(1 \mathrm{H}, \mathrm{br}) ;{ }^{13} \mathrm{CNMR}\left(62.5 \mathrm{MHz} ; \mathrm{CDCl}_{3}\right) \mathrm{d} 28.0$, $84.9,106.9,118.0,118.3,121.9,125.8,126.3,129.1,135.8,159.4 ; \mathrm{m} / \mathrm{z} 295\left(\mathrm{M}^{+}, 5 \%\right) 239$ (15), 195 (20), 115 (10), 57 (100), 41 (40), 29 (20).

6-Bromo-1-tert-butoxycarbonylindole-3-carboxamide (8). CSI (2.24 g, $15.84 \mathrm{mmol})$ was added dropwise to a solution of tert-butyl 6-bromoindole-1-carboxylate $6(2.33 \mathrm{~g}, 7.92 \mathrm{mmol})$, and acetonitrile $(18 \mathrm{~mL})$ over a $10 \mathrm{~min}$ period at $0{ }^{\circ} \mathrm{C}$. The mixture was warmed to $\mathrm{rt}$ and stirred for $1 \mathrm{~h}$. A solution of acetone $(10 \mathrm{~mL})$ and water $(2 \mathrm{~mL})$ was added and the solution was rendered alkaline by the addition of potassium hydroxide (10\% solution). The mixture was extracted with dichloromethane $(3 \times 20 \mathrm{~mL})$ and the extracts were combined and washed with brine $(20 \mathrm{~mL})$, dried $\left(\mathrm{MgSO}_{4}\right)$ and evaporated under reduced pressure. Flash column chromatography (light petroleum : dichloromethane elution) gave the title compound (1.69 $\mathrm{g}$, $64 \%$ ) as a colorless solid, mp 149-151 ${ }^{\circ} \mathrm{C}$; (Found: $\mathrm{M}^{+}$, 338.0246. $\mathrm{C}_{14} \mathrm{H}_{15}{ }^{79} \mathrm{BrN}_{2} \mathrm{O}_{3}$ requires $\mathrm{M}$, 338.0266); $v_{\max }\left(\mathrm{CHCl}_{3}\right) / \mathrm{cm}^{-1} 1743,1667,1371,1215,759 ;{ }^{1} \mathrm{HNMR}$ d $\left(250 \mathrm{MHz} ; \mathrm{CDCl}_{3}\right) 1.68$ $\left(9 \mathrm{H}, \mathrm{s},\left(\mathrm{CH}_{3}\right)_{3}\right), 5.93\left(2 \mathrm{H}, \mathrm{br}, \mathrm{NH}_{2}\right), 7.42(1 \mathrm{H}, \mathrm{dd}, J=8.51 .6 \mathrm{~Hz}), 7.97(1 \mathrm{H}, \mathrm{d}, J=8.5 \mathrm{~Hz}), 8.04$

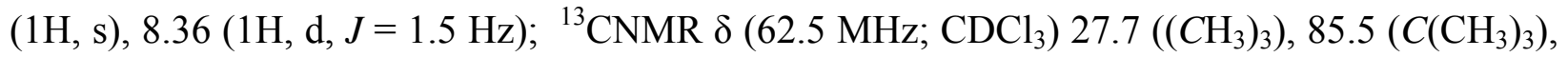
$114.9,118.1,118.8,122.4,126.3,126.8,128.5,136.2,148.3,166.2 ; \mathrm{m} / \mathrm{z} 338\left(\mathrm{M}^{+}, 5 \%\right) 282$ (10), $222(30), 114$ (15), 57 (80), 44 (90), 28 (100).

6-Bromo-1-tert-butoxycarbonylindole-3-thiocarboxamide $\quad \mathbf{( 1 0 )} \quad$ 6-Bromo-1-tertbutoxycarbonylindole-3-carboxamide 8 (300 mg, $0.88 \mathrm{mmol})$, Lawesson's reagent (179 $\mathrm{mg}, 0.44$ $\mathrm{mmol})$ and benzene $(5 \mathrm{~mL})$ were heated at reflux for $1 \mathrm{~h}$. The reaction mixture was cooled and evaporated under reduced pressure to give a yellow solid. Flash column chromatography (dichloromethane : diethyl ether elution) gave the title compound (153 $\mathrm{mg}, 49 \%$ ) as a yellow solid, mp 150-151 ${ }^{\circ} \mathrm{C}$; (Found: $\mathrm{M}^{+}$, 354.0013. $\mathrm{C}_{14} \mathrm{H}_{15}{ }^{79} \mathrm{BrNO}_{2} \mathrm{~S}$ requires M, 354.0038); $\delta \mathrm{H}$ (250 
$\left.\mathrm{MHz} ; \mathrm{CDCl}_{3}\right) 1.69\left(9 \mathrm{H}, \mathrm{s},\left(\mathrm{CH}_{3}\right)_{3}\right), 7.24(2 \mathrm{H}, \mathrm{br}, \mathrm{NH}), 7.46(1 \mathrm{H}, \mathrm{dd}, J 8.61 .7 \mathrm{~Hz}), 8.05(1 \mathrm{H}, \mathrm{d}$, $J 8.6 \mathrm{~Hz}), 8.16(1 \mathrm{H}, \mathrm{s}), 8.42$ (1H, d, J $1.7 \mathrm{~Hz}) ; \mathrm{m} / \mathrm{z} 354\left(\mathrm{M}^{+}, 50 \%\right) 220$ (80), 155 (30), 141 (45), 114 (45), $41(100)$.

Methyl 6-bromo-1-tert-butoxycarbonylindole-3-thiocarboximidate (12). 6-Bromo-1-tertbutoxycarbonylindole-3-thiocarboxamide 10 (246 $\mathrm{mg}, 0.7 \mathrm{mmol})$, methyl iodide (0.88 g, $6.2 \mathrm{mmol})$ and dichloromethane $(2 \mathrm{~mL})$ were stirred at $\mathrm{rt}$ for $48 \mathrm{~h}$ under a nitrogen atmosphere. Dichloromethane $(10 \mathrm{~mL})$ was addded and the mixture was washed with a saturated solution of sodium hydrogen carbonate $(2 \times 10 \mathrm{~mL})$, brine $(2 \times 10 \mathrm{~mL})$, dried $\left(\mathrm{MgSO}_{4}\right)$ and evaporated under reduced pressure to give a yellow gum (237 mg, 91\% crude) which was used in the next step without further purification; $v_{\max }\left(\mathrm{CHCl}_{3}\right) / \mathrm{cm}^{-1} 1742,1653,1371,1215,756 ;{ }^{1} \mathrm{HNMR}(250$ $\left.\mathrm{MHz} ; \mathrm{CDCl}_{3}\right) \delta 1.68\left(9 \mathrm{H}, \mathrm{s},\left(\mathrm{CH}_{3}\right)_{3}\right), 2.43\left(3 \mathrm{H}, \mathrm{s}, \mathrm{SCH}_{3}\right), 7.40(1 \mathrm{H}, \mathrm{dd}, J=8.4,1.7 \mathrm{~Hz}), 8.01$ $(1 \mathrm{H}, \mathrm{S}), 8.09(1 \mathrm{H}, \mathrm{d}, J=8.5 \mathrm{~Hz}), 8.35(1 \mathrm{H}, \mathrm{d}, J-=1.6 \mathrm{~Hz})$.

6-Bromo-1-tert-butoxycarbonylindole-3-carboxamidine hydrochloride (14). Methyl 6bromo-1-tert-butoxycarbonylindole-3-thiocarboximidate $12(239 \mathrm{mg}, 0.65 \mathrm{mmol})$, ammonium chloride (40 mg, $0.7 \mathrm{mmol})$ and methanol $(5 \mathrm{~mL})$ were heated under reflux for $3 \mathrm{~h}$. CAUTION: during the reaction methyl mercaptan is liberated and so a potassium permanganate bubbler was attached to the reflux condenser. The reaction mixture was cooled and ethyl acetate $(100 \mathrm{~mL})$ was added. The mixture was washed with brine $(2 \times 30 \mathrm{~mL})$, dried $\left(\mathrm{MgSO}_{4}\right)$ and evaporated under reduced pressure to give a pale brown solid, used without purification; $v_{\max }\left(\mathrm{CHCl}_{3}\right) / \mathrm{cm}^{-1}$ 1746, 1715, 1428, 1372, 1215, 759.

3-Bromoacetylindole-1-carboxylate (15). Prepared as previously described. ${ }^{7}$ tert-Butyl 3-acetyl-6-bromoindole-1-carboxylate (18). $N$, $N$-Dimethylacetamide $(0.8 \mathrm{~mL}$, $8.4 \mathrm{mmol})$ was added dropwise to a solution of phosphorus oxychloride $(0.78 \mathrm{~mL}, 8.4 \mathrm{mmol})$ and chloroform $(10 \mathrm{~mL})$ maintaining the temperature below $10{ }^{\circ} \mathrm{C}$. The mixture was stirred for $10 \mathrm{~min}$ then a solution of 6-bromoindole $16(500 \mathrm{mg}, 2.56 \mathrm{mmol})$ in chloroform $(10 \mathrm{~mL})$ was added dropwise over a 30 min period, maintaining the temperature below $10{ }^{\circ} \mathrm{C}$. The mixture was then stirred at reflux for $3 \mathrm{~h}$. The mixture was cooled and potassium hydroxide $(40 \%$ solution, $50 \mathrm{~mL})$ was added. The mixture was extracted with dichloromethane $(3 \mathrm{x} 50 \mathrm{~mL})$, the extracts were combined and washed with brine $(50 \mathrm{~mL})$, dried $(\mathrm{MgSO} 4)$ and evaporated under reduced pressure to give 3-acetyl-6-bromoindole 17 as a dark brown solid. A solution of di-tertbutyl dicarbonate (408 mg, $2.8 \mathrm{mmol})$, DMAP (30 mg, $0.24 \mathrm{mmol})$ and acetonitrile $(10 \mathrm{~mL})$ was added and the mixture stirred at $\mathrm{rt}$ for $1 \mathrm{~h}$. Saturated sodium hydrogen carbonate solution $(20 \mathrm{~mL})$ was added and the mixture stirred for a further $30 \mathrm{~min}$. The mixture was extracted with diethyl ether $(2 \times 40 \mathrm{~mL})$ the extracts were combined, washed with brine $(2 \times 20 \mathrm{~mL})$, dried $\left(\mathrm{MgSO}_{4}\right)$ and evaporated under reduced pressure. Flash column chromatography (light petroleum : diethyl ether elution) gave the title compound (345 $\mathrm{mg}, 40 \%$ over 2 steps) as a colorless solid, mp 150-152 ${ }^{\circ} \mathrm{C}$; (Found: $\mathrm{M}^{+}, 337.0063 . \mathrm{C}_{15} \mathrm{H}_{16}{ }^{79} \mathrm{BrNO}_{3}$ requires M, 337.0314); $v_{\max }\left(\mathrm{CHCl}_{3}\right) / \mathrm{cm}^{-1} 1745,1668,1432,1215,756 ;{ }^{1} \mathrm{HNMR} \delta\left(250 \mathrm{MHz} ; \mathrm{CDCl}_{3}\right) 1.72(9 \mathrm{H}, \mathrm{s}$, $\left.\left(\mathrm{CH}_{3}\right)_{3}\right), 2.56\left(3 \mathrm{H}, \mathrm{s}, \mathrm{CH}_{3}\right), 7.46(1 \mathrm{H}, \mathrm{dd}, J=8.51 .8 \mathrm{~Hz}), 8.17(1 \mathrm{H}, \mathrm{s}), 8.24(1 \mathrm{H}, \mathrm{d}, J=8.5 \mathrm{~Hz})$, $8.36(1 \mathrm{H}, \mathrm{d}, J=1.8 \mathrm{~Hz}) ;{ }^{13} \mathrm{CNMR} \delta\left(62.5 \mathrm{MHz} ; \mathrm{CDCl}_{3}\right) 27.6\left(\mathrm{CH}_{3}\right), 27.9\left(\left(\mathrm{CH}_{3}\right)_{3}\right), 86.0($ 
$\left.\mathrm{C}\left(\mathrm{CH}_{3}\right)_{3}\right), 118.1,119.3,120.9,123.8,126.5,127.6,132.3,142.8,159.4,182.7 ; \mathrm{m} / \mathrm{z} 337\left(\mathrm{M}^{+}, 5 \%\right)$ 203 (20), 159 (30), 144 (60), 57 (100), 41 (30), 28 (90).

tert-Butyl 6-bromo-3-bromoacetylindole-1-carboxylate (19). tert-Butyl 3-acetyl-6-bromoindole-1carboxylate $18(254 \mathrm{mg}, 0.75 \mathrm{mmol})$, chloroform $(2 \mathrm{~mL})$ and ethyl acetate $(2 \mathrm{~mL})$ were stirred at $75^{\circ} \mathrm{C}$ under a nitrogen atmosphere for $20 \mathrm{~min}$. Copper(II)bromide $(311 \mathrm{mg}, 1.4 \mathrm{mmol}$ ) was added and the mixture was stirred at $75{ }^{\circ} \mathrm{C}$ for a further $3 \mathrm{~h}$. The mixture was cooled and filtered, washed with ethyl acetate $(30 \mathrm{~mL})$, the filtrate and washings were combined, washed with brine $(20 \mathrm{~mL})$, dried $\left(\mathrm{MgSO}_{4}\right)$ and evaporated under reduced pressure. Flash column chromatography (light petroleum : dichloromethane elution) gave the title compound (140 mg, 45\%) as a pale yelow solid, $\mathrm{mp} 180$ $181{ }^{\circ} \mathrm{C}$; (Found: $\mathrm{M}^{+}, 414.9442 . \mathrm{C}_{15} \mathrm{H}_{15}{ }^{79} \mathrm{Br}_{2} \mathrm{NO}_{3}$ requires $\left.\mathrm{M}, 414.9420\right) ; v_{\max }\left(\mathrm{CHCl}_{3}\right) / \mathrm{cm}^{-1} 1742$, 1666, 1543, 1371, 1215, 759; ${ }^{1} \mathrm{HNMR}\left(250 \mathrm{MHz} ; \mathrm{CDCl}_{3}\right)$ d $1.73\left(9 \mathrm{H}, \mathrm{s},\left(\mathrm{CH}_{3}\right)_{3}\right), 4.33(2 \mathrm{H}, \mathrm{s}$, $\left.\mathrm{CH}_{2} \mathrm{Br}\right), 7.45(1 \mathrm{H}, \mathrm{dd}, J=8.5,1.7 \mathrm{~Hz}), 8.17(1 \mathrm{H}, \mathrm{d}, J=8.5 \mathrm{~Hz}), 8.26(1 \mathrm{H}, \mathrm{s}), 8.31(1 \mathrm{H}, \mathrm{d}, J=$

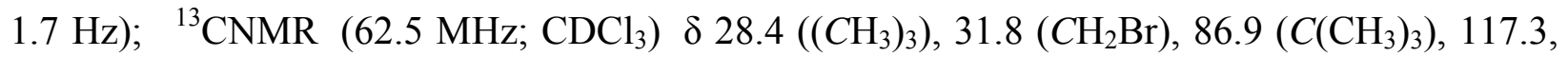
$118.7,120.11,124.1,126.5,132.8,136.4,137.9,148.7,187.4 ; \mathrm{m} / \mathrm{z} 416\left(\mathrm{M}^{+}, 5 \%\right) 317$ (10), 224 (20), 155 (20), 113 (20), 57 (100), 41 (35), 28 (80).

2-(1-tert-Butyoxycarbonylindol-3-yl)-4-(1-indol-3-yl)-1H-imidazole (20). 1-tert-Butoxycarbonylindole3-carboxamidine hydrochloride $13(92 \mathrm{mg}, 0.31 \mathrm{mmol}), 3$-bromoacetyindole 15 (75 mg, $0.31 \mathrm{mmol})$, potassium carbonate $(43 \mathrm{mg}, 0.31 \mathrm{mmol})$ and acetonitrile $(10 \mathrm{~mL})$ were heated under reflux for $7 \mathrm{~h}$. The reaction mixture was cooled and water $(10 \mathrm{~mL})$ added. The mixture was extracted with dichloromethane (2 $\mathrm{x} 10 \mathrm{~mL})$, the extracts were combined, washed with brine $(10 \mathrm{~mL})$, dried $\left(\mathrm{MgSO}_{4}\right)$ and evaporated under reduced pressure. Flash column chromatography (light petroleum : diethyl ether elution) gave the title compound (51 mg, 41\%) as an amber solid, mp 112-114 ${ }^{\circ} \mathrm{C}$; (Found: $\mathrm{M}^{+}, 398.1734$. $\mathrm{C}_{24} \mathrm{H}_{22} \mathrm{~N}_{4} \mathrm{O}_{2}$ requires $\left.\mathrm{M}, 398.1743\right) ; v_{\max }\left(\mathrm{CHCl}_{3}\right) / \mathrm{cm}^{-1} 2330$ (br), 1741, 1427, 1353, 1215, 756; ${ }^{1} \mathrm{HNMR}\left(250 \mathrm{MHz} ; \mathrm{CDCl}_{3}\right) \delta 1.55\left(9 \mathrm{H}, \mathrm{s}, \mathrm{C}\left(\mathrm{CH}_{3}\right)_{3}\right), 7.17(4 \mathrm{H}, \mathrm{m}), 7.23(3 \mathrm{H}, \mathrm{m}), 7.46(1 \mathrm{H}, \mathrm{br}$, $\mathrm{NH}), 7.76(1 \mathrm{H}, \mathrm{d}, J=8.5 \mathrm{~Hz}), 7.92(1 \mathrm{H}, \mathrm{s}), 8.09(1 \mathrm{H}, \mathrm{dd}, J=8.52 .3 \mathrm{~Hz}), 8.68(1 \mathrm{H}, \mathrm{br}, \mathrm{NH})$; ${ }^{13} \mathrm{CNMR}\left(62.5 \mathrm{MHz} ; \mathrm{CDCl}_{3}\right) \delta 27.9,84.1,108.4,111.4,111.6,115.0,119.6,120.0,120.8$, $121.8,122.2,123.2,123.6,124.8,125.1,125.2,127.5,135.2,136.3,141.1,149.4,161.3 ; \mathrm{m} / \mathrm{z} 398$ $\left(\mathrm{M}^{+},>5 \%\right) 217$ (10), 186 (10), 155 (15), 113 (15), 70 (10), 56 (50), 41 (100).

2-(6-Bromo-1-tert-butyoxycarbonylindol-3-yl)-4-(1-indol-3-yl)-1H-imidazole (21). 6-Bromo1-tert-butoxycarbonylindole-3-carboxamidine hydrochloride 14 (56 mg, $0.15 \mathrm{mmol}$ ), 3bromoacetylindole $15(36 \mathrm{mg}, 0.15 \mathrm{mmol})$, potassium carbonate $(21 \mathrm{mg}, 0.15 \mathrm{mmol})$ and acetonitrile $(5 \mathrm{~mL})$ were heated under reflux for $14 \mathrm{~h}$. The reaction mixture was cooled and water $(10 \mathrm{~mL})$ added. The mixture was extracted with dichloromethane $(2 \times 10 \mathrm{~mL})$, the extracts were combined, washed with brine $(10 \mathrm{~mL})$, dried $\left(\mathrm{MgSO}_{4}\right)$ and evaporated under reduced pressure. Flash column chromatography (light petroleum : diethyl ether elution) gave the title compound (24 mg, 34\%) as a pale brown solid, which turned dark brown over a few days; mp 145-147 ${ }^{\circ} \mathrm{C}$; (Found: $\mathrm{M}^{+}, 476.0828 . \mathrm{C}_{24} \mathrm{H}_{21}{ }^{79} \mathrm{BrN}_{4} \mathrm{O}_{2}$ requires 478.0848); $v_{\max }\left(\mathrm{CHCl}_{3}\right) / \mathrm{cm}^{-1} 3230$ (br), 1739 , 1427, 1371, 1310, 1215, 756; ${ }^{1} \mathrm{HNMR}\left(250 \mathrm{MHz} ; \mathrm{CDCl}_{3}\right) \delta 1.59\left(9 \mathrm{H}, \mathrm{s}, \mathrm{C}\left(\mathrm{CH}_{3}\right)_{3}\right), 7.21(4 \mathrm{H}$, m), $7.43(1 \mathrm{H}, \mathrm{s}), 4.46(1 \mathrm{H}, \mathrm{d}, J=3.4 \mathrm{~Hz}), 7.58(1 \mathrm{H}, \mathrm{br}, \mathrm{NH}), 7.87(1 \mathrm{H}, \mathrm{s}), 7.92(1 \mathrm{H}, \mathrm{dd}, J=8.5$, 
$3.2 \mathrm{~Hz}), 8.09(1 \mathrm{H}, \mathrm{d}, J=8.5 \mathrm{~Hz}), 8.33(1 \mathrm{H}, \mathrm{s}), 8.51(1 \mathrm{H}, \mathrm{br}, \mathrm{NH}) ; \mathrm{m} / \mathrm{z} 478\left(\mathrm{M}^{+}\left({ }^{81} \mathrm{Br}\right),>5 \%\right), 479$ $\left(\mathrm{M}^{+}\left({ }^{79} \mathrm{Br}\right),>5 \%\right), 267$ (20), 217 (20), 186 (20), 155 (40), 113 (40), 70 (25), 51 (100), 31 (60).

\section{Acknowledgements}

We thank the U.K. Cancer Research Campaign for support of this work.

\section{References}

1. Sakemi, S.; Sun, H.H. J. Org. Chem. 1991, 56, 4304.

2. Kaswasaki, I.; Yamashita, M.; Ohta, S. Chem. Commun. 1994, 2085.

3. Kaswasaki, I.; Yamashita, M.; Ohta, S. Chem. Pharm. Bull. 1996, 44, 1931.

4. Gu, X. H.; Wan, X. Z.; Jiang, B. Bioorg. Med. Chem. Lett. 1999, 9, 569.

5. Moody, C. J.; Swann, E. J. Chem. Soc., Perkin Trans. 1 1993, 2561.

6. Moody, C. J.; Swann, E.; Houlbrook, S.; Stephens, M. A.; Stratford, I. J. J. Med. Chem. 1995, 38, 1039.

7. Moody, C. J.; Roffey, J. R. A.; Stephens, M. A.; Stratford, I. J. Anti-Cancer Drugs 1997, 8, 489.

8. Doyle, K. J.; Moody, C. J. Synthesis 1994, 1021.

9. Vorbrüggen, H. Tetrahedron Lett. 1968, 1631.

10. Bergman, J.; Bäckvall, J. E.; Lindström, J.-O. Tetrahedron 1973, 29, 971.

11. Kosower, E. M.; Wu, G. S. J. Org. Chem. 1963, 28, 633. 